\title{
Hepatectomy for spontaneous rupture of hepatocellular carcinoma without portal triad clamping
}

\author{
ZHI-MING WANG ${ }^{1}$, XIN-SHENG LU ${ }^{1}$ and FU QIU ${ }^{2}$ \\ ${ }^{1}$ Department of General Surgery, Xiangya Hospital, Central South University, Changsha 410008; \\ ${ }^{2}$ Department of General Surgery, Xiangya Third Hospital, Central South University, Changsha 410013, P.R. China
}

Received May 8, 2016; Accepted June 9, 2017

DOI: $10.3892 / \mathrm{ol} .2017 .6654$

\begin{abstract}
Hepatectomy without portal triad clamping may decrease the incidence of liver injury; however, the effects of hepatectomy without portal triad clamping in the treatment of spontaneous rupture of hepatocellular carcinoma (SRHCC) remain unclear. The aims of the present study were to evaluate the therapeutic value of hepatectomy without portal triad clamping in the treatment of patients with SRHCC. The present study retrospectively reviewed patients with SRHCC who received hepatectomy without portal triad clamping (non-clamping group) and the therapeutic efficacy was compared with that of 20 patients with SRHCC undergoing the same surgery in the presence of portal triad clamping (clamping group). Following hepatectomy, the non-clamping group exhibited a significantly lower incidence of acute liver failure compared with the clamping group $(\mathrm{P}<0.05)$. No significant differences in operative time, intra-operative blood loss, disease-free or overall survival times between the two groups were identified (all $\mathrm{P}>0.05$ ). At 1 week and 2 weeks after surgery, the non-clamping group exhibited significantly lower alanine aminotransferase, aspartate aminotransferase and total bilirubin serum levels compared with the clamping group (all $\mathrm{P}<0.05$ ). Hepatectomy without portal triad clamping may decrease the incidence of liver injury and liver failure in patients with SRHCC, suggesting that it may be a safe and effective therapeutic strategy.
\end{abstract}

Correspondence to: Dr Fu Qiu, Department of General Surgery, Xiangya Third Hospital, Central South University, 138 Tongzipo Road, Changsha 410013, P.R. China

E-mail: quuffu@hotmail.com

\begin{abstract}
Abbreviations: SRHCC, spontaneous rupture of hepatocellular carcinoma; HCC, hepatocellular carcinoma; ALT, alanine aminotransferase; AST, aspartate aminotransferase; TBil, total bilirubin; Hb, hemoglobin; PT, prothrombin time; KPTT, kaolin partial thromboplastin time; AFP, $\alpha$-fetoprotein; PLT, platelets; FBI, fibrinogen; TAE, transcatheter arterial embolization; TACE, transcatheter arterial chemoembolization
\end{abstract}

Key words: hepatectomy, portal triad clamping, spontaneous rupture

\section{Introduction}

Spontaneous rupture of hepatocellular carcinoma (SRHCC) is a rare but fatal complication observed in patients with hepatocellular carcinoma (HCC). The incidence of SRHCC in patients with $\mathrm{HCC}$ is between 3 and $15 \%$, and such patients exhibit a poor therapeutic outcome. In addition, the mortality rate of patients with SRHCC is currently between 50 and $80 \%(1,2)$. A previous study of 12 patients with SRHCC who received a hepatectomy, observed that 1 patient (8.3\%) with Child-Pugh class B survived for only 4 days and succumbed to liver failure [the diagnosis of liver failure was according to the American Association for the Study of Liver Diseases (3)]; the remaining 11 patients $(91.7 \%$ ) had a median survival time of 16.5 months (4). Hepatectomy for patients with SRHCC prolongs their mean survival time; however, there have been instances of post-operative liver failure leading to mortality. Potential reasons for this may be: First, patients with SRHCC experience various extents of blood loss prior to surgery, which may result in hemodynamic changes or even decreased hepatic blood supply; secondly, liver ischemia/reperfusion as a result of surgery and portal triad clamping may further aggravate liver injury; and lastly, concomitant hepatic cirrhosis also predicts a poor prognosis (5). The prognosis of patients with SRHCC may therefore be improved if liver injury is attenuated and the risk of liver failure following surgery is minimized. Between January 1970 and February 2010, a total of 38 patients with SRHCC received a hepatectomy at Xiangya Hospital and Xiangya Third Hospital (Changsha, China), and 18 patients received a hepatectomy without portal triad clamping which resulted in attenuation of liver injury and decreased short-term mortality.

\section{Patients and methods}

Patients. A total of 38 patients with SRHCC underwent a hepatectomy at Xiangya Hospital and Xiangya Third Hospital between January 1970 and February 2010. The present study was approved by the Ethics Committee of Xiangya Third Hospital and informed written consent was obtained from each patient. Patients that were treated with sorafenib, transcatheter arterial embolization (TAE) or transcatheter arterial chemoembolization (TACE) were excluded. The sample comprised 31 male and 7 female patients ranging between 22 and 
68 years of age. Non-coagulable blood was observed following abdominal paracentesis, and postoperative pathology revealed HCC, confirming the diagnosis of SRHCC. The following criteria were used for the selection of patients with SRHCC for emergency hepatectomy: i) Patients were in a good general condition of health prior to SRHCC; ii) shock [systolic blood pressure $\leq 70 \mathrm{mmHg}$ or systolic blood pressure between 71 and $90 \mathrm{mmHg}$ with heart rate $\geq 108$ beats per min (6)] was rapidly controlled shortly following admission; iii) hepatic cirrhosis was mild during the surgery; iv) cancer was confined to a single lobe and clinicians had no difficulty in performing the hepatectomy; and v) there were no metastatic foci.

Grouping. In the present retrospective study, patients were divided into two groups on the basis of hepatic portal triad clamping during surgery (the clamping group and non-clamping group).

The portal triad clamping maneuver was routinely used with cycles of clamp/unclamp times of $15 / 5 \mathrm{~min}$. There were 20 patients in the clamping group including 16 males and 4 females. The median age was 51 years (range, 22-60 years). A total of 14 patients in this group (70\%) had a history of hepatitis B and 2 patients had a concomitant Schistosoma infection. All 20 patients exhibited abdominal pain, 14 patients $(70 \%)$ had generalized peritonitis and 7 patients (35\%) had shock. Notably, 16 patients (80\%) had serum AFP > 200 U/1. All 20 patients underwent ultrasonography and 11 patients received computed tomography scans. The two procedures revealed space-occupying lesions in the liver. Concomitant hepatic cirrhosis was noted in 14 patients (70\%), Child-Pugh class $\mathrm{A}$ in 16 patients $(80 \%)$ and Child-Pugh class $\mathrm{B}$ in 4 patients (20\%) according to the Child-Pugh classification (7). A total of 19 patients $(95 \%)$ exhibited a single cancer and 1 patient $(5 \%)$ exhibited multiple foci in the liver. The diameter of tumors ranged between 6.0 and $10.5 \mathrm{~cm}$ (mean, $8 \mathrm{~cm}$ ).

There were 18 patients in the non-clamping group, including 15 males and 3 females. The median age was 56 years (range, 26-68 years). A total of 15 patients (83.3\%) had a history of hepatitis B (HBV) and 1 patient had a concomitant Schistosoma infection. All 18 patients exhibited abdominal pain, 13 patients (72.2\%) exhibited signs of generalized peritonitis and 7 patients $(38.9 \%)$ had shock. Notably, 15 patients $(83.3 \%)$ had serum AFP $>200 \mathrm{U} / 1$. A total of 11 patients underwent ultrasonography and 16 patients received computed tomography scans. The two procedures revealed space-occupying lesions in the liver and ascites. Concomitant hepatic cirrhosis was noted in 14 patients (77.8\%), Child-Pugh class A in 12 patients $(66.7 \%)$ and Child-Pugh class B in 6 patients (33.3\%). A total of 16 patients $(88.9 \%)$ had a single cancer, whereas 2 patients (11.1\%) had multiple foci in the liver. The diameter of tumors ranged between 7.0 and $12.5 \mathrm{~cm}$ (mean, $9 \mathrm{~cm}$ ). There were no marked differences in clinical characteristics, Child-Pugh classification or cancer diameter between the two groups (Table I).

\section{Therapeutic protocol}

Clamping group. The hepatic portal triad was clamped at room temperature for a mean of $9 \mathrm{~min}$ (range, 2-22 min). Of the 20 patients, 19 received an emergency hepatectomy and peritoneal lavage. Of the patients who received an emergency hepatectomy, 8 patients received a left lateral lobectomy, 2 patients received a left inner lobectomy and 1 patient received a left hepatectomy. A total of 3 patients received a regional HCC resection and 5 patients received a partial right hepatectomy. All 20 patients had hemostasis with gauzes and peritoneal lavage due to lack of a blood source, with a second left hepatectomy performed $36 \mathrm{~h}$ later. Intra-operative pathology revealed that the surgical margin was negative in patients who received regional HCC resections. Oxygen inhalation, fluid supplementation and administration of albumin and broad-spectrum antibiotics were performed post-operatively. Patients treated later than the year 2000 received oral lamivudine (100 mg per day) following surgery when HBV DNA levels were $>500$ copies $/ \mathrm{ml}$.

Non-clamping group. Hepatectomy was performed without portal triad clamping. All 18 patients received an emergency hepatectomy and peritoneal lavage. A total of 6 patients received a left lateral lobectomy, 2 patients received a left inner lobectomy and 2 patients received a left hepatectomy. A total of 4 patients received a regional HCC resection and 4 patients received a partial right hepatectomy. Intra-operative pathology revealed that the surgical margin was negative in patients who received regional hepatectomy. Intra-operative peritoneal lavage and other post-operative therapies were identical with those in the clamping group.

Observations and clinical evaluations. The intra-operative blood loss, volume of blood transfusion, surgical time, incidence of post-operative complications and mortality were compared between the two groups.

Fresh liver specimens were obtained during surgery and examined immediately by pathologists with hematoxylin and eosin (HE) staining. Hemoglobin (Hb), platelets (PLT), alanine aminotransferase (ALT), aspartate aminotransferase (AST), total bilirubin (TBil), fibrinogen (FBI) and $\alpha$-fetoprotein (AFP) levels were determined, and prothrombin (PT) and kaolin partial thromboplastin (KPTT) times were recorded prior to, and at 1 week and 2 weeks after, hepatectomy. Ultrasonography and/or computed tomography scans were used for imaging the liver prior to, and at 2 weeks after, hepatectomy. The liver tissue samples collected during surgery were processed for pathological examination.

Follow-up. All the patients received follow-up examinations. The disease-free and overall survival times were determined.

Statistical analysis. Quantitative data are shown as the mean \pm standard deviation. Quantitative data obtained were compared among the groups by one-way analysis of variance followed by Tukey's post-hoc test. Qualitative data were analyzed using a $\chi^{2}$ test and survival analysis was calculated using the Kaplan-Meier estimator method with a log-rank test. $\mathrm{P}<0.05$ was considered to indicate a statistically significant difference.

\section{Results}

Evaluation of intra-operative blood loss, blood transfused, surgery time, mortality and incidence of post-operative complications. In the clamping group, intra-operative blood 
Table I. Preoperative data for patients undergoing hepatectomy with or without portal triad clamping.

\begin{tabular}{|c|c|c|c|}
\hline Parameter & Clamping group $n=20$ & Non-clamping group $n=18$ & P-value \\
\hline Mean age (range), years & $51(22-60)$ & $56(26-68)$ & NS \\
\hline Sex & & & NS \\
\hline Male & 16 & 15 & \\
\hline Female & 4 & 3 & \\
\hline Mean tumor size (range), $\mathrm{cm}$ & $8(6-10.5)$ & $9(7-12.5)$ & NS \\
\hline Number of tumors & & & NS \\
\hline Single & 19 & 16 & \\
\hline Multiple & 1 & 2 & \\
\hline Hemoglobin, g/l & $83.4 \pm 8.4$ & $78.3 \pm 7.7$ & NS \\
\hline Platelet count, $\times 10^{9} / 1$ & $109.7 \pm 9.3$ & $89.2 \pm 10.5$ & NS \\
\hline Child-Pugh class & & & NS \\
\hline $\mathrm{A}$ & 16 & 12 & \\
\hline $\mathrm{B}$ & 4 & 6 & \\
\hline With liver cirrhosis & 14 & 14 & NS \\
\hline HBsAg-positive & 14 & 15 & NS \\
\hline
\end{tabular}

NS, not significant; HBsAg, hepatitis B surface antigen.

loss was between 50 and $3,625 \mathrm{ml}$, the volume of blood transfused was between 100 and $4,200 \mathrm{ml}$, and the operative time was between 90 and $280 \mathrm{~min}$. Surgical complications observed among the clamping group included acute liver failure (5 patients), gastrointestinal bleeding (1 patient), lung infection ( 3 patients) and wound infection (4 patients). In the clamping group, 1 patient succumbed to acute liver failure 4 days after surgery (mortality rate of 5\%). In the non-clamping group, the intra-operative blood loss was between 100 and $2,900 \mathrm{ml}$, volume of blood transfused was between 0 and $3,000 \mathrm{ml}$, and the operative time was between 90 and $320 \mathrm{~min}$. Surgical complications in this group included lung infection (1 patient) and wound infection (2 patients). All these patients recovered following non-surgical therapies and acute liver failure; gastrointestinal bleeding and mortality were not observed (Table II). The non-clamping group exhibited a significantly lower incidence of acute liver failure compared with the clamping group $(\mathrm{P}<0.05)$, but no significant differences in blood loss, volume of blood transfused, operative time or mortality rate between the two groups were identified (all $\mathrm{P}>0.05$ ).

Blood levels of $H b, P L T, A L T, A S T, T B i l, P T, K P T T, F B I$ and $A F P$. No significant differences were observed in $\mathrm{Hb}$, PLT, ALT, AST, TBil, PT, KPTT, FBI or AFP between the two groups prior to surgery (all P>0.05). Serum ALT, AST and TBil levels in the non-clamping group were significantly decreased at 1 week and 2 weeks after surgery compared with the clamping group (all $\mathrm{P}<0.05$ ), but no significant differences in $\mathrm{Hb}$, PLT, PT, KPTT, PFIB or AFP levels between the two groups were observed (all $\mathrm{P}>0.05$; Fig. 1).

Liver imaging examinations. Prior to surgery, computed tomography scans of the liver in the two groups revealed space-occupying lesions surrounded by multiple high-intensity hematomas and hemoperitoneum (Fig. 2A). At 2 weeks after surgery, computed tomography scans of the liver revealed no space-occupying lesions in the liver and surrounding hematomas, and hemoperitoneum were not observed (Fig. 2B).

Pathological examination. Pathological examination of specimens from the two groups revealed HCC with hemorrhagic necrosis and hematoma (Fig. 3A), as well as venous rupture and thrombi at the site of rupture (Fig. 3B).

Disease-free survival and overall survival. Of the 38 patients with SRHCC (97.4\%), 37 received an emergency hepatectomy, these patients did not exhibit intra-operative peritoneal dissemination. The 1-, 3- and 5-year survival rates were 67.6 $(25 / 37), 24.3(9 / 37)$ and $16.2 \%$ (6/37), respectively. In addition, $2.6 \%(1 / 38)$ of the patients with SRHCC received a two-stage liver resection and exhibited no intra-operative peritoneal dissemination. However, these patients succumbed to peritoneal dissemination of liver cancer 9 months later (Fig. 4A).

In the clamping group, the survival time ranged from 4 days to 309 months (median, 17.0 months) and the mean disease-free survival time was 12.5 months. The 1-, 3- and 5-year survival rates were 65.5 (13/20), $25.0(5 / 20)$ and $15.0 \%$ $(3 / 20)$, respectively. In the non-clamping group, the median survival time was 28.5 months (range, 1.5-294 months) and the mean disease-free survival time was 18.25 months. The 1-, 3 - and 5-year survival rates were 6.7 (12/18), 22.2 (4/18) and $16.7 \%$ (3/18), respectively. There were no significant differences in disease-free or overall survival rates between the two groups ( $\mathrm{P}>0.05$; Fig. 4B).

\section{Discussion}

Surgical therapy of SRHCC remains contentious and certain clinicians recommend non-surgical therapy including TAE 
Table II. Operative data for undergoing hepatectomy with or without portal triad clamping.

\begin{tabular}{|c|c|c|c|}
\hline Parameter & Clamping group $n=20$ & Non-clamping group $n=18$ & P-value \\
\hline Mean operative time (range), min & $189.5(90-280)$ & $194.4(90-320)$ & NS \\
\hline Mean blood loss (range), $\mathrm{ml}$ & $989.8(50-3,625)$ & $1061.0(100-2,900)$ & NS \\
\hline Mean blood transfusion (range), ml & $921.0(100-4,200)$ & $895.0(0-3,000)$ & NS \\
\hline \multicolumn{4}{|l|}{ Treatment } \\
\hline Emergency hepatectomy & 19 & 18 & NS \\
\hline Staged hepatectomy & 1 & 0 & NS \\
\hline \multicolumn{4}{|l|}{ Type of resection } \\
\hline Left lateral lobectomy & 8 & 6 & NS \\
\hline Left lobe resection & 2 & 2 & NS \\
\hline Left liver resection & 2 & 2 & NS \\
\hline Limited & 3 & 4 & NS \\
\hline Partial right hepatectomy & 5 & 4 & NS \\
\hline \multicolumn{4}{|l|}{ Complication } \\
\hline Total & 13 & 3 & 0.0026 \\
\hline Gastrointestinal bleeding & 1 & 0 & NS \\
\hline Pulmonary infection & 3 & 1 & NS \\
\hline Acute liver failure & 5 & 0 & 0.0228 \\
\hline Wound infection & 4 & 2 & NS \\
\hline
\end{tabular}

NS, not significant.

and TACE. The hemostasis rate of TAE was as high as $97 \%$ $(32 / 33)$ and the therapeutic efficacy of TAE suggests that it may be an emergent strategy for hemostasis. However, patients receiving TAE have a median survival time of 9 weeks (8). TACE has been demonstrated to effectively inhibit the growth of HCC cells and decrease tumor size; the hemostasis rate was as high as $100 \%$ in the short term (24/24) and patients receiving TACE may survive for longer than 110 days (9). Although TAE and TACE are minimally invasive and exhibit a high hemostasis rate, the survival time of patients receiving TAE or TACE was decreased when compared with patients receiving hepatectomy. The presence of SRHCC does not reflect that the cancer is in an advanced stage and HCC not progressing into the advanced stage is associated with the risk of spontaneous rupture (5). Additionally, since a number of clinical studies have suggested that hepatectomy for SRHCC may prolong the survival time, certain clinicians preferentially perform a hepatectomy to treat patients with SRHCC (10-14).

When to surgically intervene in SRHCC treatment remains controversial and it remains unclear whether emergency hepatectomy has an improved outcome compared with TAE followed by two-stage hepatectomy. Clinicians who support TAE followed by hepatectomy propose that TAE is effective for hemostasis in the acute stage of SRHCC and may subsequently allow more time for evaluation of $\mathrm{HCC}$, and improvement of hepatic functional reservation before two-stage hepatectomy $(15,16)$. A previous study revealed that patients receiving TAE for hemostasis and subsequent two-stage hepatectomy exhibited 1-, 3- and 5-year survival rates of 88.5, 64.4 and $55.2 \%$, respectively (17). Clinicians supporting emergency hepatectomy suggested that SRHCC may cause abdominal dissemination of HCC cells due to bleeding (18). SRHCC has been revealed to cause abdominal metastasis (19-21) and multiple metastatic foci have been observed 3 months after SRHCC (22). However, intra-operative peritoneal lavage may effectively prevent abdominal metastasis of HCC, delay the recurrence of $\mathrm{HCC}$ following surgery and improve the survival rate (23). In addition, the progression of HCC may cause metastasis due to a long interval between TAE and hepatectomy, and the untreated hemoperitoneum may increase the risk of ankylenteron and sepsis. It has been reported that $10 \%$ of patients with SRHCC receiving two-stage hepatectomy developed intra-operative abdominal dissemination and $33.3 \%$ developed recurrence during the postoperative follow-up. However, no abdominal dissemination was observed in patients with SRHCC receiving emergency hepatectomy and $20 \%$ of these patients exhibited recurrence during the postoperative follow-up. Investigating clinicians therefore recommended that hepatectomy and peritoneal lavage be performed as early as possible to decrease the incidence of abdominal dissemination (24). In the present study, 97.4\% of patients received emergency hepatectomy, intra-operative peritoneal lavage and negative surgical margins confirmed by intra-operative pathological examination. Intra-operative peritoneal dissemination was not noted, and the 1-, 3- and 5-year survival rates were 67.6 (25/37), 24.3 (9/37) and $16.2 \%$ (6/37), respectively. The survival rates in these patients were consistent with those of previous studies $(1,23)$. Emergency hepatectomy may achieve definite hemostasis, treat HCC and decrease abdominal dissemination. Emergency hepatectomy, subsequent peritoneal lavage and negative surgical margins, confirmed by intra-operative pathological examination, may 

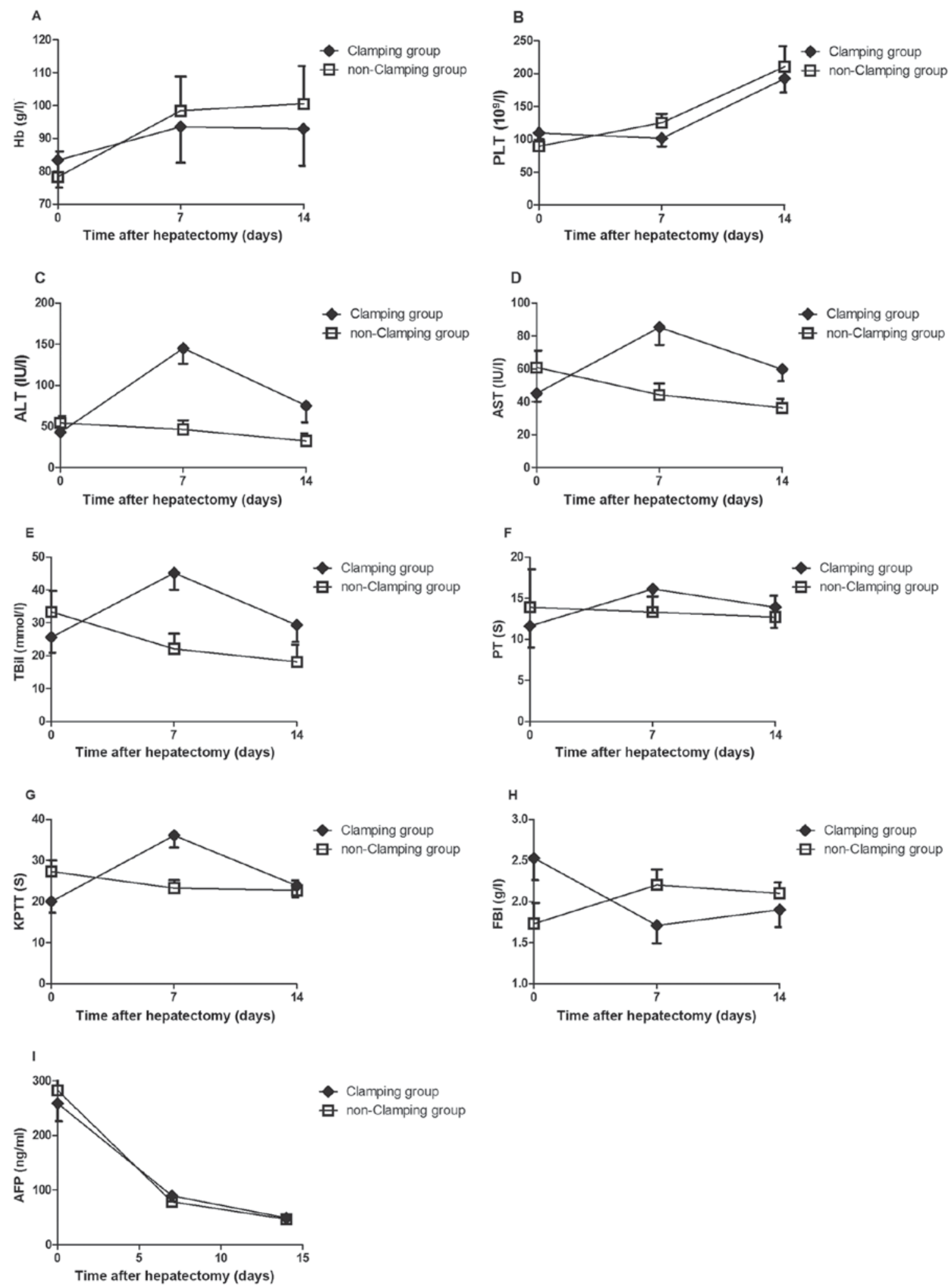

Figure 1. Levels of (A) Hb, (B) PLT, (C) ALT, (D) AST, (E) TBil, (F) PT, (G) KPTT, (H) FBI and (I) AFP prior to, and 7 and 14 days after, clamping or non-clamping hepatectomy (mean \pm standard deviation). At 1 week and 2 weeks after surgery, the non-clamping group exhibited significantly lower serum levels of ALT, AST and TBil compared with the clamping group (all P<0.05). Hb, hemoglobin; PLT, platelets; ALT, alanine aminotransferase; AST, aspartate aminotransferase; TBil, total bilirubin; PT, prothrombin time; KPTT, kaolin partial thromboplastin time; FBI, fibrinogen; AFP, $\alpha$-fetoprotein.

therefore be effective therapeutic strategies for SRHCC and for decreasing abdominal dissemination.

During hepatectomy, hepatic portal triad clamping is usually used to completely or partially block blood supply to the liver at room temperature, aiming to decrease blood loss during surgery and increase safety (25). However, hepatic portal triad clamping has been revealed to cause liver ischemia/reperfusion injury which may induce postoperative 

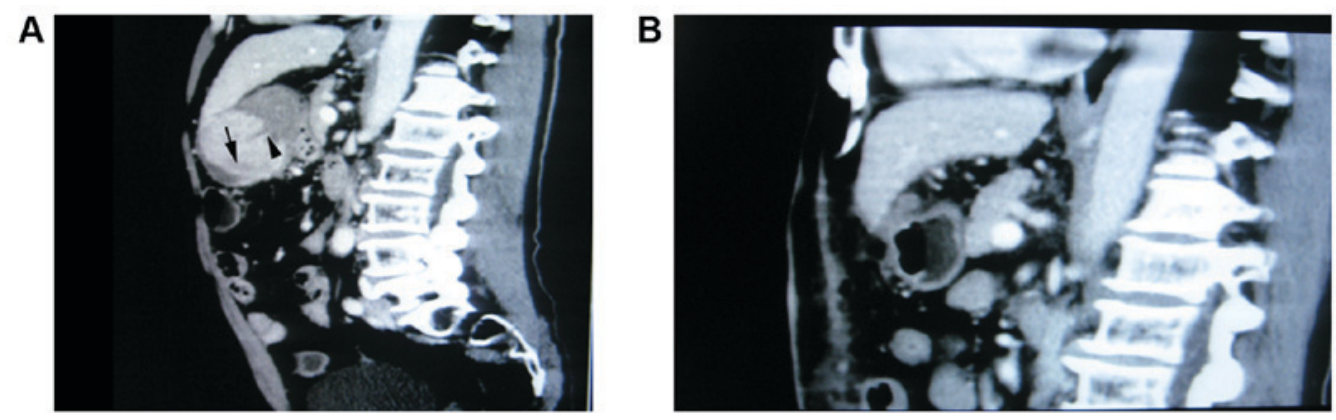

Figure 2. (A) Prior to surgery, computed tomography scans of the liver identified discontinuity of the liver tumor capsule, cancer rupture and a crescent hematoma at the site of rupture (arrowhead) and necrotic foci in cancer (arrow). (B) At 2 weeks after surgery, computed tomography scans revealed no space-occupying lesions in the liver, and surrounding hematomas and hemoperitoneum were not observed.

A

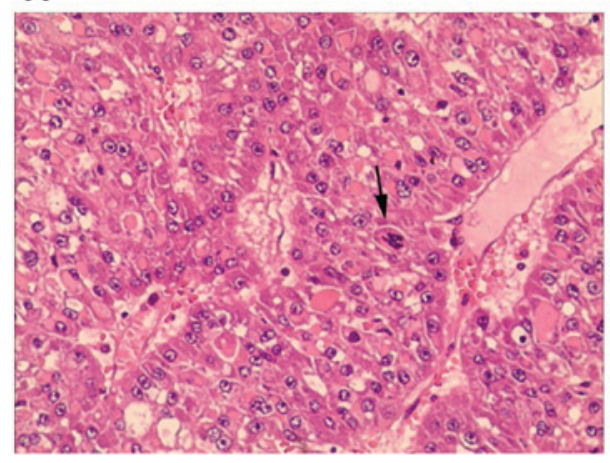

B

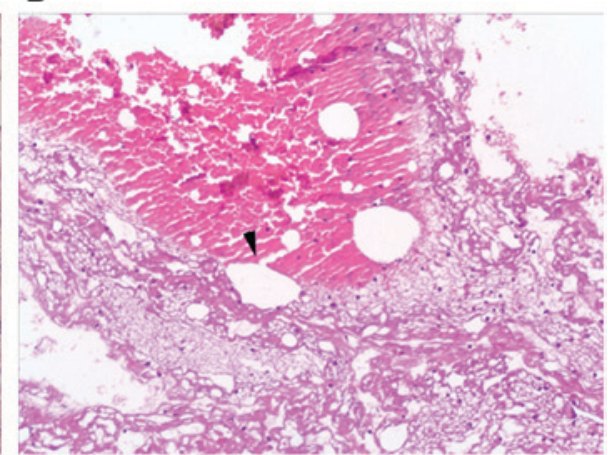

Figure 3. Pathological examination of liver specimens from clamping and non-clamping groups demonstrated: (A) Disordered hepatic cords, dark, multipolar and heteromorphic nuclei and mitose atypique identifying moderate differentiation hepatocellular carcinoma (arrow), (hematoxylin and eosin; magnification, $\mathrm{x} 100$ ); (B) discontinuity of small venous wall, venous rupture and thrombi at the site of rupture (hematoxylin and eosin; magnification, $\mathrm{x} 400$ ).

A

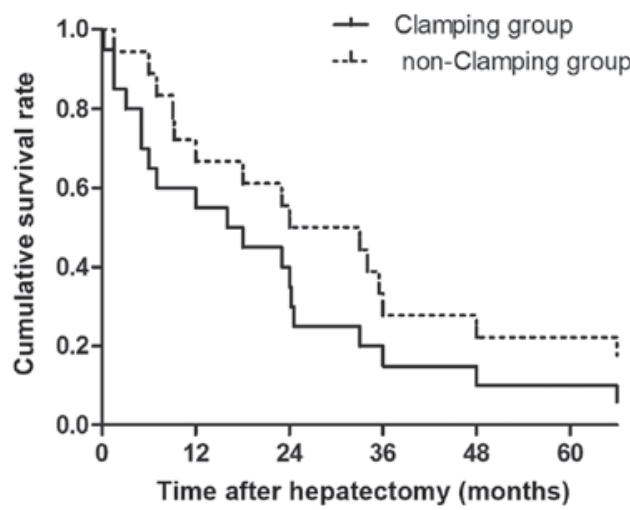

B

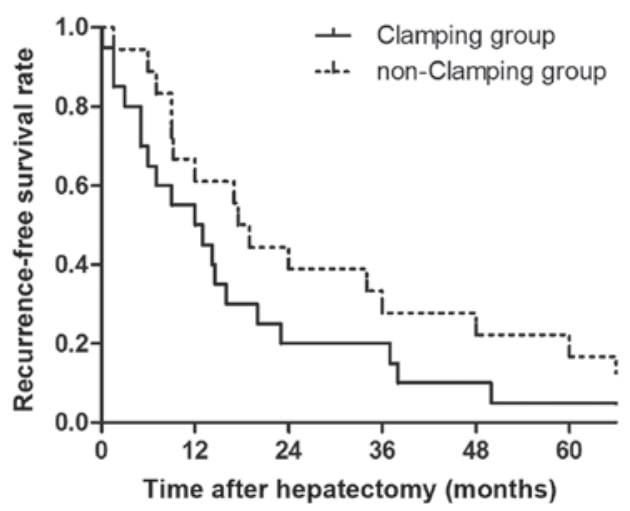

Figure 4. (A) Cumulative and (B) recurrence-free survival rates of patients with spontaneous rupture of hepatocellular carcinoma following hepatectomy with or without portal triad clamping. No significant difference in survival rate between these two groups was identified.

liver failure or even mortality (26). In certain patients, $\mathrm{HCC}$ is derived from chronic hepatic fibrosis and hepatic cirrhosis (27). In the present study, $73.7 \%$ (28/38) of patients with SRHCC had concomitant hepatic cirrhosis. However, the cirrhotic liver is susceptible to transient ischemia and patients with SRHCC and hepatic cirrhosis exhibited bleeding and ischemic injury before hepatectomy. The use of hepatic portal triad clamping for hepatectomy therefore comes with a risk of severe complications including liver failure and infection. Additionally, postoperative recovery is often unstable and patients are at risk of mortality after surgery. Attenuating postoperative liver injury and liver failure in patients with SRHCC and hepatic cirrhosis is of pivotal clinical importance. Although there is evidence demonstrating that hepatectomy without portal triad clamping may attenuate postoperative liver injury and decrease the incidence of liver failure (28), to the best of our knowledge, there are no clinical trial data in patients with SRHCC to 
confirm this. Patients with SRHCC usually have a poor prognosis and those who decline surgery often succumbed within 1 month (29). Hepatectomy in patients with SRHCC may significantly prolong survival time. A study evaluating 60 patients with SRHCC who received hepatectomy revealed that the 1-, 3- and 5-year survival rates were 54.2, 35.0 and $21.1 \%$, respectively. These data indicated that the prognosis of these patients was comparable with that of patients with HCC without SRHCC (72.1, 47.3 and 33.3\%, respectively) (1). However, this therapy possesses risks of postoperative liver failure and mortality following surgery. Attenuation of liver failure and decrease postoperative mortality has been a challenge in patients with SRHCC receiving hepatectomy, with portal triad clamping having been used to attenuate liver injury in patients with HCC. Clinical trials exploring the use of hepatectomy without portal triad clamping have not been reported in patients with HCC with SRHCC, a life-threatening complication of HCC.

The aim of the present study was to decrease liver injury and postoperative mortality in 18 patients with SRHCC by treating them with hepatectomy without portal triad clamping. The results of the present study demonstrate that the incidence of acute liver failure and postoperative recovery of liver function in the non-clamping group was superior to that in the clamping group, although there was no marked difference in survival time. Portal triad clamping aggravates liver injury and increases the incidence of acute liver failure. Hepatectomy without portal triad clamping attenuates liver injury and decreases the incidence of acute liver failure. The results of the present study suggest that hepatectomy without portal triad clamping is the preferred strategy to treat patients with SRHCC.

In summary, hepatectomy without portal triad clamping attenuated liver injury and decreased the incidence of acute liver failure in patients with SRHCC with hepatic cirrhosis; intra-operative peritoneal lavage and negative surgical margins confirmed by intra-operative pathological examination may increase the survival rate of patients with SRHCC.

\section{Acknowledgements}

The present study was supported by the Key Development Program of Hunan Province of China (grant no. 2015JC3003, to FQ).

\section{References}

1. Yeh CN, Lee WC, Jeng LB, Chen MF and Yu MC: Spontaneous tumour rupture and prognosis in patients with hepatocellular carcinoma. Br J Surg 89: 1125-1129, 2002.

2. Kim PT, Su JC, Buczkowski AK, Schaeffer DF, Chung SW, Scudamore $\mathrm{CH}$ and Ho SG: Computed tomography and angiographic interventional features of ruptured hepatocellular carcinoma: Pictorial essay. Can Assoc Radiol J 57: 159-168, 2006.

3. Lee WM, Stravitz RT and Larson AM: Introduction to the revised American association for the study of liver diseases position paper on acute liver failure, 2011. Hepatology 55: 965-967, 2012

4. Lü XS, Zheng YS and Fan QQ: Liver resection for spontaneous rupture of primary hepatocellular carcinoma. The Chinese-German Journal of Clinical Oncology 2: 23-24, 2003.

5. Battula N, Madanur M, Priest O, Srinivasan P, O'Grady J, Heneghan MA, Bowles M, Muiesan P, Heaton N and Rela M: Spontaneous rupture of hepatocellular carcinoma: A western experience. Am J Surg 197: 164-167, 2009.
6. Bulger EM, May S, Kerby JD, Emerson S, Stiell IG, Schreiber MA, Brasel KJ, Tisherman SA, Coimbra R, Rizoli S, et al: Out-of-hospital hypertonic resuscitation after traumatic hypovolemic shock: A randomized, placebo controlled trial. Ann Surg 253: 431-441, 2011.

7. Zhang J, Ye L, Zhang J, Lin M, He S, Mao X, Zhou X and Zhi F: MELD scores and Child-Pugh classifications predict the outcomes of ERCP in cirrhotic patients with choledocholithiasis: A retrospective cohort study. Medicine (Baltimore) 94: e433, 2015.

8. Ngan H, Tso WK, Lai CL and Fan ST: The role of hepatic arterial embolization in the treatment of spontaneous rupture of hepatocellular carcinoma. Clin Radiol 53: 338-341, 1998.

9. Kim JY, Lee JS, Oh DH, Yim YH and Lee HK: Transcatheter arterial chemoembolization confers survival benefit in patients with a spontaneously ruptured hepatocellular carcinoma. Eur J Gastroenterol Hepatol 24: 640-645, 2012.

10. Hsueh KC, Fan HL, Chen TW, Chan DC, Yu JC, Tsou SS, Chang TM and Hsieh CB: Management of spontaneously ruptured hepatocellular carcinoma and hemoperitoneum manifested as acute abdomen in the emergency room. World J Surg 36: 2670-2676, 2012.

11. Andersson R, Tranberg KG and Bengmark S: Hemoperitoneum after spontaneous rupture of liver tumor: Results of surgical treatment. HPB Surg 1: 81-83, 1988.

12. Shirabe K, Kitamura M, Tsutsui S, Maeda T, Matsumata T and Sugimachi K: A long-term survivor of ruptured hepatocellular carcinoma after hepatic resection. J Gastroenterol Hepatol 10: 351-354, 1995.

13. Chen ZY, Qi QH and Dong ZL: Etiology and management of hemorrhage in spontaneous liver rupture: A report of 70 cases. World J Gastroenterol 8: 1063-1066, 2002.

14. Marini P, Vilgrain V and Belghiti J: Management of spontaneous rupture of liver tumours. Dig Surg 19: 109-113, 2002.

15. Leung CS, Tang CN, Fung KH and Li MK: A retrospective review of transcatheter hepatic arterial embolisation for ruptured hepatocellular carcinoma. J R Coll Surg Edinb 47: 685-688, 2002.

16. Tanaka A, Takeda R, Mukaihara S, Hayakawa K, Shibata T, Itoh K, Nishida N, Nakao K, Fukuda Y, Chiba T and Yamaoka Y: Treatment of ruptured hepatocellular carcinoma. Int J Clin Oncol 6: 291-295, 2001.

17. Lee HS, Choi GH, Kang DR, Han KH, Ahn SH, Kim DY, Park JY, Kim SU and Choi JS: Impact of spontaneous hepatocellular carcinoma rupture on recurrence pattern and long-term surgical outcomes after partial hepatectomy. World J Surg 38: 2070-2078, 2014.

18. Sonoda T, Kanematsu T, Takenaka K and Sugimachi K: Ruptured hepatocellular carcinoma evokes risk of implanted metastases. J Surg Oncol 41: 183-186, 1989.

19. Okano J, Shiota G, Horie Y, Mitsuda A, Suou T, Kawasaki H and Oofuji S: Rupture of metastatic nodule on the peritoneal surface secondary to hepatocellular carcinoma. Intern Med 35: 783-784, 1996.

20. Yunoki Y, Takeuchi H, Makino Y, Murakami I, Yasui Y, Tanakaya K, Kawaguchi K and Konaga E: Intraperitoneal seeding of ruptured hepatocellular carcinoma: Case report. Abdom Imaging 24: 398-400, 1999.

21. Eriguchi N, Aoyagi S, Okuda K, Tamae T, Fukuda S, Kanazawa N, Hamada S, Kawabata M, Nishimura K and Kodama T: Successful surgical treatment for implanted intraperitoneal metastases of hepatocellular carcinoma. J Hepatobiliary Pancreat Surg 7: $520-523,2000$

22. Lin CC, Chen CH, Tsang YM, Jan IS and Sheu JC: Diffuse intraperitoneal metastasis after spontaneous rupture of hepatocellular carcinoma. J Formos Med Assoc 105: 577-582, 2006.

23. Chang YM, Hsu KF, Yu JC, Chan DC, Chen CJ, Chen TW, Hsieh $\mathrm{CB}$ and Hsieh HF: Distilled water peritoneal lavage in patients with rupture hepatocellular carcinoma. Hepatogastroenterology 60: 140-143, 2013.

24. Yang T, Sun YF, Zhang J, Lau WY, Lai EC, Lu JH, Shen F and Wu MC: Partial hepatectomy for ruptured hepatocellular carcinoma. Br J Surg 100: 1071-1079, 2013.

25. Qian NS, Liao YH, Cai SW, Raut V and Dong JH: Comprehensive application of modern technologies in precise liver resection. Hepatobiliary Pancreat Dis Int 12: 244-250, 2013.

26. Tashiro H, Kuroda S, Mikuriya Y and Ohdan $\mathrm{H}$ : Ischemia-reperfusion injury in patients with fatty liver and the clinical impact of steatotic liver on hepatic surgery. Surg Today 44: 1611-1625, 2014. 
27. Ramakrishna G, Rastogi A, Trehanpati N, Sen B, Khosla R and Sarin SK: From cirrhosis to hepatocellular carcinoma: New molecular insights on inflammation and cellular senescence. Liver Cancer 2: 367-383, 2013.

28. Wrightson WR, Edwards MJ and McMasters KM: The role of the ultrasonically activated shears and vascular cutting stapler in hepatic resection. Am Surg 66: 1037-1040, 2000.
29. Zhu Q, Li J, Yan JJ, Huang L, Wu MC and Yan YQ: Predictors and clinical outcomes for spontaneous rupture of hepatocellular carcinoma. World J Gastroenterol 18: 7302-7307, 2012. 